This item was submitted to Loughborough's Research Repository by the author.

Items in Figshare are protected by copyright, with all rights reserved, unless otherwise indicated.

\title{
Jacobi-Trudy formula for generalized Schur polynomials
}

PLEASE CITE THE PUBLISHED VERSION

http://www.mathjournals.org/mmj/2014-014-001/2014-014-001-007.html

\section{PUBLISHER}

American Mathematical Society $@$ Independent University of Moscow

\section{VERSION}

VoR (Version of Record)

\section{PUBLISHER STATEMENT}

This work is made available according to the conditions of the Creative Commons Attribution-NonCommercialNoDerivatives 4.0 International (CC BY-NC-ND 4.0) licence. Full details of this licence are available at: https://creativecommons.org/licenses/by-nc-nd/4.0/

\section{LICENCE}

CC BY-NC-ND 4.0

\section{REPOSITORY RECORD}

Sergeev, A.N., and A.P. Veselov. 2019. "Jacobi-trudy Formula for Generalized Schur Polynomials”. figshare. https://hdl.handle.net/2134/15662. 


\title{
JACOBI-TRUDY FORMULA FOR GENERALISED SCHUR POLYNOMIALS
}

\author{
A.N. SERGEEV AND A.P. VESELOV
}

\begin{abstract}
Jacobi-Trudy formula for a generalisation of Schur polynomials related to any sequence of orthogonal polynomials in one variable is given. As a corollary we have Giambelli formula for generalised Schur polynomials.
\end{abstract}

To Grisha Olshanski on his 65-th birthday with admiration

\section{INTRODUCTION}

The classical Jacobi-Trudy formula expresses the Schur polynomials

$$
S_{\lambda}\left(x_{1}, \ldots, x_{n}\right)=\frac{\left|\begin{array}{cccc}
x_{1}^{\lambda_{1}+n-1} & x_{2}^{\lambda_{1}+n-1} & \ldots & x_{n}^{\lambda_{1}+n-1} \\
x_{1}^{\lambda_{2}+n-2} & x_{2}^{\lambda_{2}+n-2} & \ldots & x_{n}^{\lambda_{2}+n-2} \\
\vdots & \vdots & \ddots & \vdots \\
x_{1}^{\lambda_{n}} & x_{2}^{\lambda_{n}} & \ldots & x_{n}^{\lambda_{n}}
\end{array}\right|}{\Delta_{n}(x)}
$$

where $\lambda=\left(\lambda_{1}, \ldots, \lambda_{n}\right)$ is a partition and $\Delta_{n}(x)=\prod_{i<j}^{n}\left(x_{i}-x_{j}\right)$, as the determinant

$$
S_{\lambda}\left(x_{1}, \ldots, x_{n}\right)=\left|\begin{array}{cccc}
h_{\lambda_{1}} & h_{\lambda_{1}+1} & \ldots & h_{\lambda_{1}+l-1} \\
h_{\lambda_{2}-1} & h_{\lambda_{2}} & \ldots & h_{\lambda_{2}+l-2} \\
\vdots & \vdots & \ddots & \vdots \\
h_{\lambda_{l}-l+1} & h_{\lambda_{l}-l+2} & \ldots & h_{\lambda_{l}}
\end{array}\right|
$$

where $l=l(\lambda)$ and $h_{i}=h_{i}\left(x_{1}, \ldots, x_{n}\right)$ are the complete symmetric polynomials (see [1]). Note that these polynomials $h_{i}$ are particular case of Schur polynomials $S_{\lambda}$, corresponding to the partition $\lambda=(i)$ consisting of one part.

In this note we give a version of this formula, which is valid for the following generalisation of Schur polynomials related to any sequence of orthogonal polynomials in one variable.

More precisely, let $\left\{\varphi_{i}(z)\right\}, i=0,1,2, \ldots$ be a sequence of polynomials in one variable, which satisfy a three-term recurrence relation

$$
z \varphi_{i}(z)=\varphi_{i+1}(z)+a(i) \varphi_{i}(z)+b(i) \varphi_{i-1}(z)
$$

with $\varphi_{0} \equiv 1, \varphi_{-1} \equiv 0$ (for example, a sequence of the orthogonal polynomials $[2])$. The corresponding generalised Schur polynomials $S\left(x_{1}, \ldots, x_{n} \mid a, b\right)$ are 
defined for any partition $\lambda$ and two infinite sequences $a=\left\{a_{i}\right\}, b=\left\{b_{i}\right\}$ by the Weyl-type formula

$$
S_{\lambda}\left(x_{1}, \ldots, x_{n} \mid a, b\right)=\frac{\left|\begin{array}{cccc}
\varphi_{\lambda_{1}+n-1}\left(x_{1}\right) & \varphi_{\lambda_{1}+n-1}\left(x_{2}\right) & \ldots & \varphi_{\lambda_{1}+n-1}\left(x_{n}\right) \\
\varphi_{\lambda_{2}+n-2}\left(x_{1}\right) & \varphi_{\lambda_{2}+n-2}\left(x_{2}\right) & \ldots & \varphi_{\lambda_{2}+n-2}\left(x_{n}\right) \\
\vdots & \vdots & \ddots & \vdots \\
\varphi_{\lambda_{n}}\left(x_{1}\right) & \varphi_{\lambda_{n}}\left(x_{2}\right) & \ldots & \varphi_{\lambda_{n}}\left(x_{n}\right)
\end{array}\right|}{\Delta_{n}(u)} .
$$

For $n=1$ we assume that $\Delta_{1} \equiv 1$, so for $\lambda=(i)$ we have $S_{\lambda}\left(x_{1} \mid a, b\right)=$ $\varphi_{i}\left(x_{1}\right)$. If the initial sequence $\varphi_{i}(z)$ was orthogonal with measure $d \mu(z)$ the polynomials $S_{\lambda}\left(x_{1}, \ldots, x_{n} \mid a, b\right)$ are orthogonal with respect to the measure

$$
\Omega(z)=\Delta_{n}^{2}(z) \prod_{i=1}^{n} d \mu\left(z_{i}\right) .
$$

Alternatively, the generalised Schur polynomials can be defined in this case as the polynomials of the triangular form

$$
S_{\lambda}\left(x_{1}, \ldots, x_{n} \mid a, b\right)=\sum_{\mu \preceq \lambda} K_{\lambda, \mu}(a, b) m_{\mu}\left(x_{1}, \ldots, x_{n}\right),
$$

which are orthogonal with respect to the measure (5). Here $m_{\mu}\left(x_{1}, \ldots, x_{n}\right)$ are monomial symmetric polynomials [1] and the notation $\mu \preceq \lambda$ means that $\mu_{1}+\cdots+\mu_{k} \leq \lambda_{1}+\cdots+\lambda_{k}$ for all $1 \leq k \leq n$.

When $\varphi_{i}(z)$ is the sequence of classical Jacobi polynomials [2], the corresponding generalised Schur polynomials coincide with the multidimensional Jacobi polynomials with parameter $\theta=1$ (see Lassalle [4] and OkounkovOlshanski [5]).

Denote the polynomials $S_{\lambda}\left(x_{1}, \ldots, x_{n} \mid a, b\right)$ with $\lambda=(i, 0, \ldots, 0)$ as $h_{i}(x)$ and extend this sequence for negative $i$ by assuming that $h_{i}(x) \equiv 0$ for $i<0$. Extend also the sequence of coefficients $a(i)$ and $b(i)$ to the negative $i$ arbitrarily and define recursively the polynomials $h_{i}^{(r)}\left(x_{1}, \ldots, x_{n}\right)$ by the relation

$$
h_{i}^{(r+1)}=h_{i+1}^{(r)}+a(i+n-1) h_{i}^{(r)}+b(i+n-1) h_{i-1}^{(r)}
$$

with initial data $h_{i}^{(0)}(x)=h_{i}(x)$. One can check that $h_{i}^{(r)}(x) \equiv 0$ whenever $i+r<0$ and that the definition of the polynomials $h_{i}^{(r)}\left(x_{1}, \ldots, x_{n}\right)$ does not depend on the extension of the coefficients to the negative $i$ provided

$$
r \leq i+2 n-2 \text {. }
$$

In particular, all the entries of the formula (9) below are well defined.

Our main result is the following 
Theorem 1.1. The generalised Schur polynomials satisfy the following JacobiTrudy formula:

$$
S_{\lambda}\left(x_{1}, \ldots, x_{n} \mid a, b\right)=\left|\begin{array}{cccc}
h_{\lambda_{1}} & h_{\lambda_{1}}^{(1)} & \ldots & h_{\lambda_{1}}^{(l-1)} \\
h_{\lambda_{2}-1} & h_{\lambda_{2}-1}^{(1)} & \ldots & h_{\lambda_{2}-1}^{(l-1)} \\
\vdots & \vdots & \ddots & \vdots \\
h_{\lambda_{l}-l+1} & h_{\lambda_{l}-l+1}^{(1)} & \ldots & h_{\lambda_{l}-l+1}^{(l-1)}
\end{array}\right|
$$

where $l=l(\lambda)$.

This gives a universal proof of the Jacobi-Trudy and Giambelli formulas for usual Schur polynomals as well as for the characters of symplectic and orthogonal Lie algebras (see [3]) and for the factorial Schur polynomials $[1,6]$. Another interesting case, which seems to be new, is the Jacobi-Trudy formula for the multidimensional Jacobi polynomials with parameter $\theta=1$.

\section{PROOF}

We start with the following lemma.

Lemma 2.1. The following equality holds

$$
h_{i}^{(r)}\left(x_{1}, \ldots, x_{n}\right)-x_{1} h_{i}^{(r-1)}\left(x_{1}, \ldots, x_{n}\right)=h_{i+1}^{(r-1)}\left(x_{2}, \ldots, x_{n}\right)
$$

for all $r, i$ satisfying the relation (8).

Proof. The proof is by induction in $r$. When $r=1$ we have from definition

$$
\begin{aligned}
& h_{i}^{(1)}\left(x_{1}, \ldots, x_{n}\right)-x_{1} h_{i}^{(0)}\left(x_{1}, \ldots, x_{n}\right)=h_{i+1}\left(x_{1}, \ldots, x_{n}\right)+a(i+n-1) h_{i}\left(x_{1}, \ldots, x_{n}\right) \\
& +b(i+n-1) h_{i-1}\left(x_{1}, \ldots, x_{n}\right)-x_{1} h_{i}\left(x_{1}, \ldots, x_{n}\right)= \\
& =\Delta_{n}(x)^{-1}\left|\begin{array}{cccc}
0 & \left(x_{2}-x_{1}\right) \varphi_{i+n-1}\left(x_{2}\right) & \ldots & \left(x_{n}-x_{1}\right) \varphi_{i+n-1}\left(x_{n}\right) \\
\varphi_{n-2}\left(x_{1}\right) & \varphi_{n-2}\left(x_{2}\right) & \ldots & \varphi_{n-2}\left(x_{n}\right) \\
\vdots & \vdots & \ddots & \vdots \\
1 & 1 & \ldots & 1
\end{array}\right| .
\end{aligned}
$$

Subtracting the first column from the others we get

$$
\begin{gathered}
=\Delta_{n}(x)^{-1}\left|\begin{array}{cccc}
0 & \left(x_{2}-x_{1}\right) \varphi_{i+n-1}\left(x_{2}\right) & \ldots & \left(x_{n}-x_{1}\right) \varphi_{l+n-1}\left(x_{n}\right) \\
\varphi_{n-2}\left(x_{1}\right) & \varphi_{n-2}\left(x_{2}\right)-\varphi_{n-2}\left(x_{1}\right) & \ldots & \varphi_{n-2}\left(x_{n}\right)-\varphi_{n-2}\left(x_{1}\right) \\
\vdots & \vdots & \ddots & \vdots \\
1 & 0 & \ldots & 0
\end{array}\right|= \\
=\Delta_{n-1}(x)^{-1}\left|\begin{array}{ccc}
\varphi_{i+n-1}\left(x_{2}\right) & \ldots & \varphi_{i+n-1}\left(x_{n}\right) \\
\frac{\varphi_{n-2}\left(x_{2}\right)-\varphi_{n-2}\left(x_{1}\right)}{x_{2}-x_{1}} & \ldots & \frac{\varphi_{n-2}\left(x_{n}\right)-\varphi_{n-2}\left(x_{1}\right)}{x_{n}-x_{1}} \\
\vdots & \ddots & \vdots \\
\frac{\varphi_{1}\left(x_{2}\right)-\varphi_{1}\left(x_{1}\right)}{x_{2}-x_{1}} & \ldots & \frac{\varphi_{1}\left(x_{n}\right)-\varphi_{1}\left(x_{1}\right)}{x_{n}-x_{1}}
\end{array}\right|=h_{i+1}\left(x_{2}, \ldots, x_{n}\right) .
\end{gathered}
$$

The induction step is straightforward check using the relation (7). 
Now we are ready to prove the Jacobi-Trudy formula. The proof is by induction in $l=l(\lambda)$. If $l=1$ then the formula follows from the definition of $h_{i}$. Suppose that $l>1$. We will use the bracket $\left\{g\left(x_{1}, \ldots, x_{n}\right)\right\}$ to denote the result of the alternation:

$$
\left\{g\left(x_{1}, \ldots, x_{n}\right)\right\}=\sum_{w \in S_{n}} \varepsilon(w) g\left(x_{w(1)}, \ldots, x_{w(n)}\right) .
$$

We claim that

$$
\left\{h_{i}^{(r)}\left(x_{1}, \ldots, x_{n}\right) x_{1}^{n-1} x_{2}^{n-2} \ldots x_{n}^{0}\right\}=\left\{x_{1}^{r} \varphi_{i+n-1}\left(x_{1}\right) x_{2}^{n-2} \ldots x_{n}^{0}\right\}
$$

for any $r \leq i+2 n-2$. Indeed, for $r=0$ this true by definition and the induction step follows easily from relations (3) and (7). From this we have

$$
\left.\begin{array}{c}
\left\{\left|\begin{array}{cccc}
h_{\lambda_{1}} & h_{\lambda_{1}}^{(1)} & \ldots & h_{\lambda_{1}}^{(l-1)} \\
h_{\lambda_{2}-1} & h_{\lambda_{2}-1}^{(1)} & \ldots & h_{\lambda_{2}-1}^{(l-1)} \\
\vdots & \vdots & \ddots & \vdots \\
h_{\lambda_{l}-l+1} & h_{\lambda_{l}-l+1}^{(1)} & \ldots & h_{\lambda_{l}-l+1}^{(l-1)}
\end{array}\right| x_{1}^{n-1} x_{2}^{n-2} \ldots x_{n}^{0}\right\} \\
=\left\{\begin{array}{cccc}
\varphi_{\lambda_{1}+n-1}\left(x_{1}\right) & x_{1} \varphi_{\lambda_{1}+n-1}\left(x_{1}\right) & \ldots & x_{1}^{l-1} \varphi_{\lambda_{1}+n-1}\left(x_{1}\right) \\
h_{\lambda_{2}-1} & h_{\lambda_{2}-1}^{(1)} & \ldots & h_{\lambda_{2}-1}^{(l-1)} \\
\vdots & \vdots & \ddots & \vdots \\
h_{\lambda_{l}-l+1} & h_{\lambda_{l}-l+1}^{(1)} & \ldots & h_{\lambda_{l}-l+1}^{(l-1)}
\end{array} \mid x_{2}^{n-2} \ldots x_{n}^{0}\right.
\end{array}\right\} .
$$

Multiply every column except the last one by $x_{1}$ and subtract it from the next column. Then by lemma this expression is equal to

$$
\begin{aligned}
& \left\{\left|\begin{array}{cccc}
\varphi_{\lambda_{1}+n-1}\left(x_{1}\right) & 0 & \ldots & 0 \\
h_{\lambda_{2}-1} & \hat{h}_{\lambda_{2}-1} & \ldots & \hat{h}_{\lambda_{2}-1}^{(l-2)} \\
\vdots & \vdots & \ddots & \vdots \\
h_{\lambda_{l}-l+1} & \hat{h}_{\lambda_{l}-l+1} & \ldots & \hat{h}_{\lambda_{l}-l+1}^{(l-2)}
\end{array}\right| x_{2}^{n-2} \ldots x_{n}^{0}\right\} \\
& =\left\{\varphi_{\lambda_{1}+n-1}\left(x_{1}\right)\left|\begin{array}{ccc}
\hat{h}_{\lambda_{2}-1} & \ldots & \hat{h}_{\lambda_{2}-1}^{(l-2)} \\
\vdots & \ddots & \vdots \\
\hat{h}_{\lambda_{l}-l+1} & \ldots & \hat{h}_{\lambda_{l}-l+1}^{(-2)}
\end{array}\right| x_{2}^{n-2} \ldots x_{n}^{0}\right\},
\end{aligned}
$$

where $\hat{h}_{i}^{(r)}=h_{i}^{(r)}\left(x_{2}, \ldots, x_{n}\right)$. By induction this is equal to

$$
\left\{\varphi_{\lambda_{1}+n-1}\left(x_{1}\right) \varphi_{\lambda_{2}+n-2}\left(x_{2}\right) \ldots \varphi_{\lambda_{n}}\left(x_{n}\right)\right\},
$$

which by definition coincides with $f_{\lambda}$. This completes the proof of the main theorem. 


\section{Giambelli formula}

As a corollary we have the following Giambelli formula for generalised Schur functions. ${ }^{1}$ Let us denote the generalised Schur polynomials corresponding to the hook Young diagrams as

$$
S_{(u \mid v)}(x)=S_{\left(u+1,1^{(v)}\right)}(x) .
$$

Theorem 3.1. The generalised Schur polynomials satisfy the following Giambelli formula

$$
S_{\lambda}\left(x_{1}, \ldots, x_{n} \mid a, b\right)=\left|\begin{array}{cccc}
S_{\left(\lambda_{1}-1 \mid \lambda_{1}^{\prime}-1\right)} & S_{\left(\lambda_{1}-1 \mid \lambda_{2}^{\prime}-2\right)} & \ldots & S_{\left(\lambda_{1}-1 \mid \lambda_{r}^{\prime}-r\right)} \\
S_{\left(\lambda_{2}-2 \mid \lambda_{1}^{\prime}-1\right)} & S_{\left(\lambda_{2}-2 \mid \lambda_{2}^{\prime}-2\right)} & \ldots & S_{\left(\lambda_{2}-2 \mid \lambda_{r}^{\prime}-r\right)} \\
\vdots & \vdots & \ddots & \vdots \\
S_{\left(\lambda_{r}-r \mid \lambda_{1}^{\prime}-1\right)} & S_{\left(\lambda_{r}-r \mid \lambda_{2}^{\prime}-2\right)} & \ldots & S_{\left(\lambda_{r}-r \mid \lambda_{r}^{\prime}-r\right)}
\end{array}\right|
$$

where $r$ is the number of the diagonal boxes of $\lambda$.

Proof. The proof follows the same line as Macdonald's proof of the usual Giambelli formula (see [1], Ch.1, Section 3, Example 21), but we give the proof here for the reader's convenience.

From the Theorem 1.1 we see that

$$
S_{(u \mid v)}(x)=\left|\begin{array}{cccc}
h_{u+1} & h_{u+1}^{(1)} & \ldots & h_{u+1}^{(v)} \\
h_{0} & h_{0}^{(1)} & \ldots & h_{0}^{(v)} \\
\vdots & \vdots & \ddots & \vdots \\
h_{1-v} & h_{1-v}^{(1)} & \ldots & h_{1-v}^{(v)}
\end{array}\right|
$$

In this formula $u \geq 0, v \geq 0$, but we can define the functions $S_{(u \mid v)}(x)$ by the same formula for all integers $u$ and nonnegative integers $v$. It is easy to check that this defines them correctly and that for $u$ negative $S_{(u \mid v)}(x)=0$ except when $u+v=-1$, in which case $S_{(u \mid v)}(x)=(-1)^{v}$.

Now consider the following matrix of the size $j \times(j+1)$

$$
H^{(j)}=\left(\begin{array}{cccc}
h_{0} & h_{0}^{(1)} & \ldots & h_{0}^{(j)} \\
h_{-1} & h_{-1}^{(1)} & \ldots & h_{-1}^{(j)} \\
\vdots & \vdots & \ddots & \vdots \\
h_{1-j} & h_{1-j}^{(1)} & \ldots & h_{1-j}^{(j)}
\end{array}\right)
$$

and denote by $\Delta_{i}^{(j)}, 1 \leq i \leq j+1$ the determinant of its sub-matrix without the $i$-th column multiplied by $(-1)^{i-1}$. If $i>j+1$ we set by definition $\Delta_{i}^{(j)}=0$. One can check also that $\Delta_{k}^{(k-1)}=(-1)^{k-1}$.

\footnotetext{
${ }^{1}$ We are very grateful to $\mathrm{G}$. Olshanski, who pointed out this to us.
} 
For any partition $\lambda$ consider the matrices

$$
A=\left(\begin{array}{cccc}
h_{\lambda_{1}} & h_{\lambda_{1}}^{(1)} & \ldots & h_{\lambda_{1}}^{(l-1)} \\
h_{\lambda_{2}-1} & h_{\lambda_{2}-1}^{(1)} & \ldots & h_{\lambda_{2}-1}^{(l-1)} \\
\vdots & \vdots & \ddots & \vdots \\
h_{\lambda_{l}-l+1} & h_{\lambda_{l}-l+1}^{(1)} & \ldots & h_{\lambda_{l}-l+1}^{(l-1)}
\end{array}\right), B=\left(\begin{array}{cccc}
\Delta_{1}^{(l-1)} & \Delta_{1}^{(l-2)} & \ldots & \Delta_{1}^{(0)} \\
\Delta_{2}^{(l-1)} & \Delta_{2}^{(l-2)} & \ldots & \Delta_{2}^{(0)} \\
\vdots & \vdots & \ddots & \vdots \\
\Delta_{l}^{(l-1)} & \Delta_{l}^{(l-2)} & \ldots & \Delta_{l}^{(0)}
\end{array}\right)
$$

Note that $B$ is upper-triangular with respect to the anti-diagonal with the anti-diagonal elements $(-1)^{k-1}$, so the determinant of $B$ is identically equal to 1 , while the determinant of $A$ by Theorem 1.1 coincides with $S_{\lambda}\left(x_{1}, \ldots, x_{n} \mid a, b\right)$. From linear algebra and definition of $S_{(u \mid v)}$ we have

$$
A B=\left(\begin{array}{cccc}
S_{\left(\lambda_{1}-1 \mid l-1\right)} & S_{\left(\lambda_{1}-1 \mid l-2\right)} & \cdots & S_{\left(\lambda_{1}-1 \mid 0\right)} \\
S_{\left(\lambda_{2}-2 \mid l-1\right)} & S_{\left(\lambda_{2}-2 \mid l-2\right)} & \ldots & S_{\left(\lambda_{2}-2 \mid 0\right)} \\
\vdots & \vdots & \ddots & \vdots \\
S_{\left(\lambda_{l}-l \mid l-1\right)} & S_{\left(\lambda_{l}-l \mid l-2\right)} & \cdots & S_{\left(\lambda_{l}-l \mid 0\right)}
\end{array}\right) .
$$

Taking the determinants of both sides we see that $S_{\lambda}\left(x_{1}, \ldots, x_{n} \mid a, b\right)$ equals to the determinant of the last matrix. In this matrix there are many zeros since for $k>r$ we have $\lambda_{k}-k<0$ and therefore $S_{\left(\lambda_{k}-k \mid l-j\right)}=(-1)^{l-j}$ if $\lambda_{k}-k+l-j=-1$ and 0 otherwise. This means that in the $k$-th row with $k>r$ there is only one non-zero element $(-1)^{l-j}$ with $l-j=k-\lambda_{k}-1$. This reduces the calculation of the determinant to the $r \times r$ matrix with the remaining columns having the numbers $\lambda_{j}^{\prime}-j, j=1, \ldots, r$. Indeed, for any $\lambda$ of length $l$ with $r$ boxes on the diagonal the union of two sets $\left\{k-\lambda_{k}-1\right\}, k=$ $r+1, \ldots, l$ and $\left\{\lambda_{j}^{\prime}-j\right\}, j=1, \ldots, r$ is the set $\{0,1,2, \ldots, l-1\}$ as it follows, for example, from the identity

$$
\sum_{i=1}^{l} t^{i}\left(1-t^{-\lambda_{i}}\right)=\sum_{j=1}^{r}\left(t^{\lambda_{j}^{\prime}-j+1}-t^{j-\lambda_{j}}\right)
$$

(see [1], Ch.1, Section 1, Example 4). The check of the sign completes the proof.

\section{Particular cases}

As a corollary we have the following well-known cases of the Jacobi-Trudy formula.

1. When $a(i)=b(i)=0$ for all $i \geq 0$ we have $\varphi_{i}(z)=z^{i}$ and (9) clearly coincides with the usual Jacobi-Trudy formula for Schur polynomials.

2. The characters of the orthogonal Lie algebra $s o(2 n+1)$ correspond to the case when $a(i)=0, b(i)=1$ for $i>0$ and $a(0)=-1, b(0)=0$ and the polynomials $\varphi_{i}(z)=x^{i}+x^{i-1}+\cdots+x^{-i}, z=x+x^{-1}$. Using the recurrence relation (7), having in this case the form

$$
h_{i}^{(r+1)}=\underset{6}{h_{i+1}^{(r)}}+h_{i-1}^{(r)}
$$


we can rewrite the general Jacobi-Trudy formula (9) in the form known in representation theory (see Prop. 24.33 in Fulton-Harris [3]): the character $\chi_{\lambda}$ is the determinant of the $l \times l$ matrix whose $i$-th row is

$$
\left(\begin{array}{llll}
h_{\lambda_{i}-i+1} & h_{\lambda_{i}-i+2}+h_{\lambda_{i}-i} & h_{\lambda_{i}-i+3}+h_{\lambda_{i}-i-1} \ldots h_{\lambda_{i}-i+l}+h_{\lambda_{i}+2-l}
\end{array}\right) .
$$

The same is true for the characters of the even orthogonal Lie algebra $s o(2 n)$, where $a(i)=0$ for all $i \geq 0$ and $b(i)=1$ for $i>1$ with $b(1)=2$ and $\varphi_{i}(z)=x^{i}+x^{-i}, z=x+x^{-1}$ (see Prop. 24.44 in [3]) and for the symplectic Lie algebra $\operatorname{sp}(2 n)$, when $a(i)=0, b(i)=1$ for all $i \geq 0$ and $\varphi_{i}(z)=x^{i}+x^{i-2}+\cdots+x^{-i}, z=x+x^{-1}$ (Prop. 24.22 in [3]). Note that the change of $a(0)$ and $b(1)$ does not affect the definition of the relevant $h_{i}^{(r)}$ for $r>0$.

3. The factorial Schur polynomials [6] correspond to the special case when $b_{i}=0$, so that

$$
\varphi_{i}(z)=(z-a(0))(z-a(1)) \ldots(z-a(i-1)), \quad i>0 .
$$

The Jacobi-Trudy formula for them can be found in [1], Ch.1, Section 3, Example 20.

4. For $a(i), b(i)$ given by

$$
\begin{gathered}
a(x)=-\frac{2 p(p+2 q+1)}{(2 x-p-2 q-1)(2 x-p-2 q+1)}, \\
b(x)=\frac{2 x(2 x-2 q-1)(2 x-2 p-2 q-1)(2 x-2 p-4 q-2)}{(2 x-p-2 q)(2 x-p-2 q-1)^{2}(2 x-p-2 q-2)}
\end{gathered}
$$

we have the Jacobi-Trudy formula for the multidimensional Jacobi polynomials with $k=-1$, which seems to be new.

\section{INFINITE-DIMENSIONAL AND SUPER VERSIONS}

Let us assume now that the coefficients $a(i)$ and $b(i)$ of the recurrence relation are rational functions of $i$. In that case we can define the generalised Schur functions (which are the infinite-dimensional version of $S_{\lambda}(x \mid a, b)$ ) in the following way (cf. Okounkov-Olshanski [5]).

First note that the generalised Schur polynomials (4) are the linear combination of the usual Schur polynomials

$$
S_{\lambda}\left(x_{1}, \ldots, x_{n} \mid a, b\right)=\sum_{\mu \subseteq \lambda} c_{\lambda, \mu}(n \mid a, b) S_{\mu}\left(x_{1}, \ldots, x_{n}\right),
$$

where $c_{\lambda, \mu}(n \mid a, b)$ are some rational functions of $n$. The generalised Schur functions depend on the additional parameter $d$ and defined by

$$
S_{\lambda}(x \mid a, b ; d)=\sum_{\mu \subseteq \lambda} c_{\lambda, \mu}(d \mid a, b) S_{\mu}(x),
$$


where $S_{\mu}(x)$ are the usual Schur functions [1]. They satisfy the following infinite dimensional version of the Jacobi-Trudy formula:

$$
S_{\lambda}(x \mid a, b ; d)=\left|\begin{array}{cccc}
h_{\lambda_{1}} & h_{\lambda_{1}}^{(1)} & \ldots & h_{\lambda_{1}}^{(l-1)} \\
h_{\lambda_{2}-1} & h_{\lambda_{2}-1}^{(1)} & \ldots & h_{\lambda_{2}-1}^{(l-1)} \\
\vdots & \vdots & \ddots & \vdots \\
h_{\lambda_{l}-l+1} & h_{\lambda_{l}-l+1}^{(1)} & \ldots & h_{\lambda_{l}-l+1}^{(l-1)}
\end{array}\right| .
$$

Here $l=l(\lambda), h_{i}=S_{\lambda}(x \mid a, b ; d)$ with $\lambda=(i)$ if $i \geq 0$ and $h_{i} \equiv 0$ if $i<0$, $h_{i}^{(r)}=h_{i}^{(r)}(x \mid a, b ; d)$ are defined for generic $d$ by the recurrence relation

$$
h_{i}^{(r+1)}=h_{i+1}^{(r)}+a(i+d-1) h_{i}^{(r)}+b(i+d-1) h_{i-1}^{(r)}
$$

with initial data $h_{i}^{(0)}=h_{i}$.

The generalised super Schur polynomials $S_{\mu}\left(x_{1}, \ldots, x_{n} ; y_{1}, \ldots, y_{m} \mid a, b\right)$ can be defined by the same formula (14), where the Schur functions should be replaced by the super Schur polynomials $S_{\mu}\left(x_{1}, \ldots, x_{n} ; y_{1}, \ldots, y_{m}\right)$ (see e.g. [1]) and $d$ must be specialised as the superdimension $d=n-m$ (provided the coefficients have no poles at $d=n-m$ ). Alternatively, $S_{\mu}\left(x_{1}, \ldots, x_{n} ; y_{1}, \ldots, y_{m} \mid a, b\right)$ is the image of the corresponding generalised Schur function $S_{\mu}(x \mid a, b ; d)$ under the homomorphism $\phi$ sending the power sums $p_{k} \in \Lambda$ to the super power sums $x_{1}^{k}+\cdots+x_{n}^{k}-y_{1}^{k}-\cdots-y_{m}^{k}$ with $d=n-m$. In the case of factorial Schur polynomials their super version had been introduced in a different way by Molev [8].

An important example corresponds to the sequences (12),(13). In this case the generalised Schur functions coincide with the Jacobi symmetric functions with parameter $k=-1$ (see [9]). These functions and their super versions play an important role in representation theory of the orthosymplectic Lie superalgebras [10].

Finally we would like to mention that the Jacobi-Trudy formula (9) can be rewritten in a dual form in terms of the conjugate partition in the spirit of Macdonald [1] (Ch.1, Section 3, Example 21) and Okounkov-Olshanski [7], Section 13.

\section{ACKNowledgements}

We are grateful to A. Okounkov and G. Olshanski for useful comments. This work has been partially supported by the EPSRC (grants EP/E004008/1 and EP/J00488X/1).

\section{REFERENCES}

[1] I. Macdonald Symmetric functions and Hall polynomials. 2nd edition, Oxford Univ. Press, 1995.

[2] G. Szego Orthogonal polynomials. Fourth edition. AMS Colloquium Publications, Vol. XXIII. American Mathematical Society, Providence, R.I., 1975.

[3] W. Fulton, J. Harris, Representation theory. A first course. Graduate Texts in Mathematics 129. Readings in Mathematics. Springer-Verlag, New York, 1991. 
[4] M. Lassalle Polynomes de Jacobi généralisés, C.R. Acad. Sci. Paris Sér. I Math. 312 (1991), no.6, 425-428.

[5] A. Okounkov, G. Olshanski Limits of BC-type orthogonal polynomials as the number of variables goes to infinity. Jack, Hall-Littlewood and Macdonald polynomials, 281318, Contemp. Math., 417, Amer. Math. Soc., Providence, RI, 2006.

[6] L. C. Biedenharn and J. D. Louck A new class of symmetric polynomials defined in terms of tableaux, Advances in Appl. Math. 10 (1989), 396438.

[7] A. Okounkov, G. Olshanski Shifted Schur functions. St. Petersburg Math. J. 9 (1998), no. 2, 239-300.

[8] A. Molev Factorial supersymmetric Schur functions and super Capelli identities. AMS Translations (2), Vol. 181, AMS, Providence, R.I., 1997, 109-137.

[9] A.N. Sergeev, A.P. Veselov $B C_{\infty}$ Calogero-Moser operator and super Jacobi polynomials. arXive: 0807.3858.

[10] A.N. Sergeev, A.P. Veselov Euler characters and super Jacobi polynomials. In preparation.

Department of Mathematical Sciences, Loughborough University, LoughBOROUGH LE11 3TU, UK

E-mail address: A.N.Sergeev@lboro.ac.uk

Department of Mathematical Sciences, Loughborough University, LoughBorough LE11 3TU, UK and Department of Mathematics and Mechanics, Moscow State University, Moscow, 119899, Russia

E-mail address: A.P.Veselov@lboro.ac.uk 